\title{
Paraguay: ¿Jaque Mate?
}

\section{Paraguay: Is this the Endgame?}

\author{
DIEGO ABENTE BRUN \\ International Forum for Democratic Studies
}

\begin{abstract}
RESUMEN
El año 2007 estuvo caracterizado por tres desarrollos interrelacionados. En primer lugar, se destacó el fracasado intento del presidente Duarte Frutos de modificar la cláusula constitucional que prohíbe la reelección y luego su ofensiva política para proyectar su poder a través de la recomposición del stronismo como esquema de dominación y de la imposición de sus candidatos a la Presidencia y Vicepresidencia. En segundo lugar, la agudización de las características de un modelo económico de crecimiento en los sectores de la agricultura empresarial capital-intensivo y de la ganadería de exportación simultáneamente con el estancamiento en los demás sectores y el consiguiente aumento de los niveles de pobreza y miseria. En último término, la aceleración de los tempos electorales, con las consiguientes movidas político-judiciales para impedir el triunfo de la oposición y las controvertidas elecciones internas del Partido Colorado.
\end{abstract}

Palabras clave: Alianza Patriótica por el Cambio, Luis A. Castigioni, Nicanor Duarte Frutos, elecciones internas, Partido Colorado, Partido Liberal, Fernando Lugo, Blanca Ovelar de Duarte Lino Oviedo, recomposición del stronismo.

\begin{abstract}
The year 2007 was characterized by three major and interrelated trends. In the first place, one must note the failed effort of President Duarte Frutos to modify the constitutional clause that prevents him from running for re-election and his all-out effort to reconstitute the stronista model of domination and to impose his candidates to the Presidency and the Vice-Presidency. Secondly, the deepening of the characteristics of the economic model that produced high growth in agribusiness and the livestock sector while the rest of the sectors remained stagnant and the levels of poverty and indigence increased. The final process involved the acceleration of the electoral tempo with the consequent judicial-political maneuvering and the controversial primaries of the Colorado party.
\end{abstract}

Key words: Alianza Patriótica por el Cambio, Luis A. Castigioni, Nicanor Duarte Frutos, Primary Elections, Colorado Party, Liberal Party, Fernando Lugo, Blanca Ovelar de Duarte, Lino Oviedo, Recomposition of Stronismo.

\section{INTRODUCCIÓN}

El año 2007 ha sido una vez más un año eminentemente político. Si el 2006 se caracterizó por el intento desenfrenado de lograr la modificación constitucional para hacer posible la reelección del presidente Nicanor Duarte Frutos, el 2007 fue marcado por un intento de similares características para asegurar vicariamente el mismo objetivo por la interpósita figura de su ministra de Educación Blanca Ovelar de Duarte. 
Nunca desde el apogeo del general Lino Oviedo en la década de 1990 la política nacional giró por tanto tiempo y de manera tan exclusiva, y hasta excluyente, alrededor de los intereses de una persona. Este hecho polarizó al país y también lo paralizó. De modo que si en 2006 la agenda giró alrededor de los intereses del presidente Duarte Frutos de lograr la cláusula de rerreelección a cualquier precio, la de 2007 lo hizo en torno a su intento de recomponer el stronismo como sistema de dominación e imponer sus candidatos a la Presidencia y la Vicepresidencia de la República.

La oposición, por otro lado, está jugando una carta propia que pronto se aglutinó en torno a la figura y candidatura del ex Obispo Fernando Lugo. Tras la fallida estrategia de apoyar a un sector del Partido Colorado para contrarrestar las ambiciones autoritarias de Oviedo, y la participación dividida en las elecciones de 2003 que garantizó la continuidad del Partido Colorado, en esta oportunidad la oposición está jugando una carta unitaria. A principio de 2007 inició un proceso de concertación y decantación, largo y no exento de confrontaciones, pero que al final del camino terminó conformando un nuevo escenario.

El año 2008 será crucial. A escasos cuatro meses de las cuartas elecciones generales desde el comienzo de la transición a la democracia en Paraguay, el interrogante que mejor caracteriza los acontecimientos del año 2007 es si estamos en las vísperas del juego final o del final de un juego más. ¿Asistimos acaso al juego final, o "endgame", a un jaque mate en el sentido de etapa final de una acción o proceso, es decir, a la culminación del sistema de gobierno de partido hegemónico? ¿O se trata en cambio del ya acostumbrado final del quinquenal juego iterativo que presenciamos desde 1989, la habitual rotación de una parte de la elite del Partido Colorado, la continuación del statu quo y la negociación puntual con sectores de la oposición de prebendas y canonjías a cambio de votos en el Congreso? El análisis del año que se realiza a continuación nos provee con algunas claves para aproximarnos a una respuesta.

\section{LA REPATRIMONIALIZACIÓN Y RESTRONIZACIÓN DEL GOBIERNO}

Característica central del año 2007 fue el fuerte proceso centrípeto de recomposición de la hegemonía del poder en clave partidaria y personalista. Como en ningún otro período de gobierno desde 1989, se observó una tendencia acelerada y generalizada de centralizar todo el poder en el presidente. Concomitantemente, el poder estatal asumió un carácter netamente partidario y personal y todas las responsabilidades públicas de importancia fueron adjudicadas a leales seguidores del presidente y operadores políticos, no en función a su capacidad técnica ni a su afinidad ideológica, sino exclusivamente en base a su militancia $y$ lealtad al presidente. Así prácticamente todos los presidentes de seccionales (comités políticos) de Asunción ocuparon importantes cargos públicos y lo mismo sucedió con aquellos de las principales ciudades y localidades del interior. Las más altas funciones de administración del Estado se repartieron, pues, como si fueran patrimonio privado del presidente. 
Quizás la característica más resaltante y novedosa de este proceso de repatrimonialización del sistema fue el manejo de las entidades binacionales Itaipú y Yacyretá. Como lo establece la Constitución, la Ley y el buen criterio de manejo de la cosa pública todos los gobiernos anteriores transferían los recursos de royalties y compensaciones de las binacionales al Ministerio de Hacienda y de ahí en adelante su desembolso estaba reglado por el Presupuesto General de la Nación. El Gobierno de Duarte Frutos obtuvo sin embargo que el Gobierno brasileño instruyera a Itaipú pagar a Paraguay compensaciones adicionales de entre 60 y 80 millones de dólares. Según un Informe de la Contraloría General de la nación dichos recursos, denominados Fondos Sociales, no ingresaron al Presupuesto, no fueron registrados como ingresos, no fueron controlados como egresos y fueron utilizados de manera absolutamente arbitraria por el Gobierno, año tras año. (ABC, 16 de marzo de 2008) Un intento del Congreso de obligar al Gobierno a transparentar su uso y canalizarlos vía Presupuesto fue frustrado por el Ejecutivo con la complicidad de la Corte Suprema de Justicia. El Poder Ejecutivo recurrió de inconstitucionalidad y la Corte Suprema otorgó la medida cautelar solicitada de no innovar (es decir, no aplicar el artículo en cuestión) hasta que la misma se pronuncie sobre el fondo de la cuestión. Por supuesto, hasta el momento no se ha pronunciado ni se espera que se pronuncie como ha sucedido con otros casos controversiales con lo cual la Corte aparenta no dar la razón al Ejecutivo pero en la práctica se la da. ${ }^{1}$ (La Nación, 21 de diciembre de 2006, ABC, 21 de diciembre de 2006, Corte Suprema de Justicia 2008, ABC 13 de marzo 2008).

La repatrimonialización se realizó también por los conductos tradicionales vía un sofisticado sistema de información y espionaje que le permitió al Gobierno obtener información no sólo política sino también de la mayoría de los actos de corrupción y, por lo tanto, tolerarlos, frenarlos, denunciarlos, o "anotarlos" para futuras conciliaciones de cuentas. Este sistema, montado en una primera etapa por el comandante del Regimiento Escolta Presidencial coronel Heriberto Galeano fue adaptado y probablemente debilitado cuando el mismo fue cesado debido a una denuncia de corrupción, aunque no faltaron observadores que vieron en la aceptación demasiado fácil de su destitución una jugada del Gobierno para evitar el surgimiento de un virtual Montesinos. (Una saga que comenzó en mayo de 2006, Portal Paraguayo de Noticias, 25 de mayo de 2006 y aún continúa, La Nación, 2 de enero de 2008). ${ }^{2}$

1 Otro botón de muestra de esta sibilina estrategia se dio cuando a principios de 2006 el presidente Duarte Frutos en violación de la Constitución asumió el cargo de presidente del Partido Colorado. El mismo recurrió de inconstitucionalidad y solicitó una medida cautelar. La misma le fue otorgada a las resultas de la decisión sobre el fondo de la cuestión. Desde hace dos años y medio la resolución sobre el fondo sigue en el fondo de la gaveta esperando turno. En el caso cuestión de los fondos de Itaipú tanto en 2007 (presupuesto 2006) como en 2008 (presupuesto 2007) la Corte actuó de igual manera. Ver Auto Interlocutorio $\mathrm{N}^{\mathrm{o}} 17,7$ de febrero de 2008, que hace lugar al pedido de suspensión de los efectos de los artículos 108 y 121 de la Ley 3409/07 presentado por la Entidad Binacional Itaipú.

2 “El 26 de mayo funcionarios del Gobierno allanaron la residencia del coronel Heriberto Galeano, ex comandante del Regimiento Escolta Presidencial y de la Primera División de Infantería como parte de una investigación acerca de un esquema clandestino de escucha de conversaciones telefónicas. El Fiscal declaró que gran parte de los equipos habían sido removidos del lugar debido a una filtración." Bureau of Democracy, Human Rights, and Labor, U.S. Department of State, Country Report on Human Rights Practices, 2006. Washington, DC, 6 de marzo de 2007. 
Este proceso de repatrimonialización del Estado siguió las mismos patrones stronistas. A salvo del acervo patrimonial del líder, y especialmente de sus seguidores, se dejaron dos instituciones básicas para la reproducción del sistema: El Ministerio de Hacienda y el Banco Central. Durante casi toda la dictadura de Stroessner, un militar ocupó el Ministerio de Hacienda con la firme misión de asegurar el equilibrio fiscal, condición ineludible para la perpetuación del sistema. De la misma manera, el Banco Central fue confiado a un funcionario cuya misión fue evitar a toda costa el descontrol monetario y la inflación. Ambos cumplieron su función con religiosa puntillosidad hasta poco antes de la caída del stronismo. ${ }^{3}$

De la misma manera, el presidente declaró en primer lugar al Ministerio de Hacienda "zona de exclusión partidaria" de forma de asegurar una administración que salvara su nuevo gobierno de la catástrofe fiscal y financiera heredada de la administración anterior y acentuada por la crisis económica de la región. Cuando su primer ministro de Hacienda, Dionisio Borda, intentó ir más allá de la honestidad y la eficiencia en el control de la política fiscal e incluyó en su agenda otras medidas de reforma del Estado fue prontamente forzado a renunciar. Su reemplazante, Ernst Ferdinand Bergen, dio marcha atrás en las reformas pero mantuvo la "zona de exclusión" del Ministerio. Y lo mismo sucedió con el sucesor de éste y actual ministro, César Barreto, nombrado el 30 de julio de 2007 (ABC, 30 de julio de 2007).

En el caso del Banco Central el patrón fue el mismo. Con la venida de una ex funcionaria del Fondo Monetario Internacional en 2005, al mismo momento que se forzaba la renuncia del primer ministro de Hacienda, se pretendió enviar la señal que el enfoque del Gobierno no cambiaría. Y en líneas generales se mantuvo la política de "zona de exclusión" para el Banco Central, aunque en este caso hubo y siguen habiendo serias dudas sobre la confiabilidad de los índice de inflación, que en el pasado eran incuestionables.

Estos dos fenómenos, repatrimonialización y restronización, se manifestaron con fuerza en 2007 y fueron justificados por el presidente en términos que todos los cargos del Estado están a su disposición para negociarlos en función a sus intereses pues, en sus palabras, "la política no es la actividad de distribuir bienes espirituales sino de distribuir bienes materiales" (ABC, 4 de enero de 2008). Con ello consagró en el discurso lo que ya había ocurrido en la práctica: la degradación de la institucionalidad republicana.

\section{ECONOMÍA Y SOCIEDAD}

Este fuerte proceso de repatrimonialización y restronización fue acompañado y facilitado por un patrón de evolución económico a la vez estable en términos fiscales y concentrador en términos de crecimiento. Efectivamente, el comportamiento de la economía en 2007 exhibió características similares a las de 2006: crecimiento del sector agrícola capital-

Sobre el stronismo véase por ejemplo, Diego Abente, Stronismo, Post-Stronismo, and the Prospects for Democratization in Paraguay, Working Paper \# 119 - March 1989 and Paul H. Lewis, Paraguay Under Stroessner, Chapel Hill: University of north Carolina Press, 1980. 
intensivo, recesión del sector agrícola mano de obra-intensivo, y virtual estancamiento de los demás sectores. La inflación tuvo un comportamiento muy volátil, con tasas interanuales superiores al 12\% en algunos meses pero cerrando el 2007 con 7\%, según el Banco Central, tasa inferior al 12,6\% del año anterior.

El Gobierno anunció con bombos y platillos un crecimiento del PIB del 6,4\% en 2007, el más alto de los últimos cinco años de resultados positivos. Sin embargo, datos del Banco Central indican que de dicho guarismo $4,2 \%$ corresponden al sector agrícola, y en especial a la soja que registró una producción récord de 6.525 .000 toneladas, 71\% más que en 2006. En contrapartida la producción de algodón, el principal rubro de la economía campesina familiar, se redujo en casi $42 \%$ con respecto al año anterior.

En mucho menor medida contribuyó a dicha tasa de crecimiento del PIB el crecimiento lento del sector servicios, que aportó $2,2 \%$ de la tasa registrada y electricidad, agua, construcción e impuestos que contribuyeron con un $0,7 \%$. Los demás sectores de la economía experimentaron una contracción. El sector manufacturero, por ejemplo, se contrajo en $0,2 \%$ y el ganadero en un $0,4 \%$. En síntesis, todos los sectores, con excepción de la agricultura empresarial, especialmente la de la soja, experimentaron un decrecimiento o un crecimiento inferior a la tasa de crecimiento de la población ${ }^{4}$ (Banco Central del Paraguay, 2007).

Es por ello que el argumento del buen desempeño de la economía debe ser matizado observando sus dos caras; el positivo pero altamente concentrado, y el negativo, pero altamente disperso. Es pues lógico también comprender que la mayor parte de la población, que no vive de los números producidos por la soja, encuentre agobiadora su situación económica.

Esta situación fue más grave aún en algunas de las regiones más pobres del noreste del país a raíz de una devastadora ola de incendios producto de una prolongada sequía. Los incendios han sido los peores registrados en la historia del Paraguay, abarcaron más de un millón de hectáreas y afectaron a aproximadamente 100.000 familias campesinas. A modo de comparación, la superficie del siniestro fue cinco veces mayor que la devastada en los incendios del pasado verano boreal en Grecia.

El desempeño fiscal ofrece un resultado positivo con una importante nota de cautela. El cuadro que sigue cubre todo el quinquenio del actual gobierno y muestra tendencias preocupantes. En términos de guaraníes constantes los ingresos de los últimos tres años, 2005-2007 aumentaron 10\%, mientras que los gastos se incrementaron en $15 \%$. Igualmente se nota una obvia tendencia al estancamiento de las recaudaciones, ya que después del gran aumento del año 2004, 21\% en guaraníes constantes, la tasa de crecimiento real de las recaudaciones se estancó en promedio en torno al 3\%. Esto significa que la situación puede volverse insostenible en el mediano plazo y el próximo gobierno tendrá que encarar alguna reforma tributaria. ${ }^{5}$ 
Cuadro 1: Evolución de los Ingresos Tributarios 2003-2007

\begin{tabular}{|c|c|c|c|c|c|}
\hline Gobierno Central & 2003 & 2004 & 2005 & 2006 & 2007 \\
\hline \multicolumn{6}{|c|}{ Millones de Gs Corrientes. PYG } \\
\hline Ingresos & 6.066 .457 & 7.650 .259 & 8.462 .801 & 9.597 .640 & 10.719 .232 \\
\hline$\%$ Cambio & .. & 26 & 11 & 13 & 12 \\
\hline Gastos & 6.211 .598 & 6.991 .683 & 8.077.761 & 9.323 .622 & 10.244 .698 \\
\hline$\%$ Cambio & .. & 13 & 16 & 15 & 10 \\
\hline Balance & -145.141 & 658.577 & 385.041 & 274.019 & 474.534 \\
\hline$\%$ Cambio & .. & -554 & -42 & -29 & 73 \\
\hline \multicolumn{6}{|c|}{ Millones de Gs. Constantes. PYG } \\
\hline Ingresos & 6.066 .457 & 7.332 .595 & 7.514 .607 & 7.859 .290 & 8.120 .015 \\
\hline$\%$ Cambio & .. & 21 & 2 & 5 & 3 \\
\hline Gastos & 6.211 .598 & 6.701 .365 & 7.172.707 & 7.634 .902 & 7.760 .547 \\
\hline$\%$ Cambio & .. & 8 & 7 & 6 & 2 \\
\hline Balance & -145.141 & 631.230 & 341.900 & 224.388 & 359.468 \\
\hline$\%$ Cambio & .. & -535 & -46 & -34 & 60 \\
\hline IPC, Inflación, Promedio & 14,2 & 4,3 & 7,9 & 8,4 & 8,1 \\
\hline Index, 2003=100 & 100,0 & 104,3 & 112,6 & 122,1 & 132,0 \\
\hline
\end{tabular}

Fuente: Ministerio de Hacienda y Banco Central del Paraguay.

La situación social del país refleja este patrón de evolución económica así en las áreas rurales como en las ciudades. Aproximadamente dos quintos de la población vive debajo de la línea oficial de pobreza. Por primera vez en años la Dirección de Estadística no ha publicado los datos sobre la evolución de la pobreza de la Encuesta Permanente de Hogares, un indicador probable de que los números no son los que un gobierno gustaría mostrar a las puertas de unas elecciones nacionales. ${ }^{6}$

Quizás el mejor y más dramático indicador del malestar social generalizado es el continuo crecimiento de la emigración. Sólo entre 2001 y al 2006 unas 116.000 personas emigraron, es decir, más del 2\% de la población total. En el 6,2\% de los hogares al menos un miembro emigró por motivos laborales. Con $8 \%$ de su población viviendo en el exilio económico, Paraguay es uno de los países de mayor tasa de emigración en el mundo. ${ }^{7}$

6 Esto se confirmó cuando se filtraron los datos que muestran que en 2006 (datos que se recogen en 2007) aumentó el nivel de pobreza extrema de manera dramática, del 15,5 al 20,9\% en áreas rurales y del 11,6\% al $16,6 \%$ en áreas urbanas. $\mathrm{ABC}, 13$ de febrero de 2006.

7 Juan M. Carrón, "La emigración paraguaya en el contexto de la globalización", Ponencia presentada en el 3er. Congreso Paraguayo de Población “Cambio sociodemográfico, urbanización y pobreza”. Asunción, noviembre de 2007. 
Gráfico 1: Evolución de los Ingresos Tributarios 2003-2007

(Gastos Corrientes) Paraguay: Gobierno Central
Millones de Gs. Corrientes

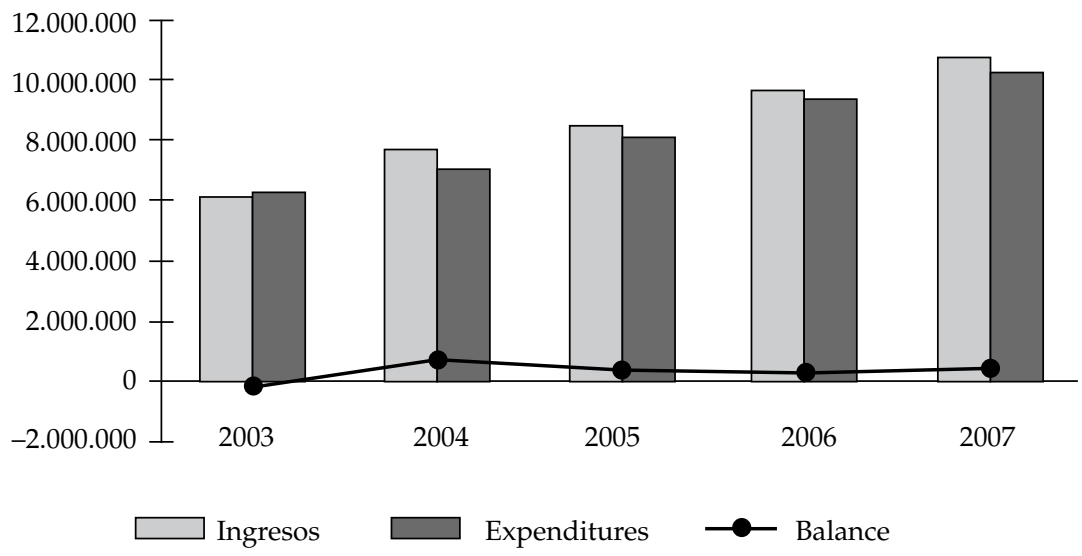

Gráfico 2: Evolución de los Ingresos Tributarios 2003-2007 (Gastos Constantes)

Paraguay: Gobierno Central

Millones de Gs. constantes PYG, $2003=100$

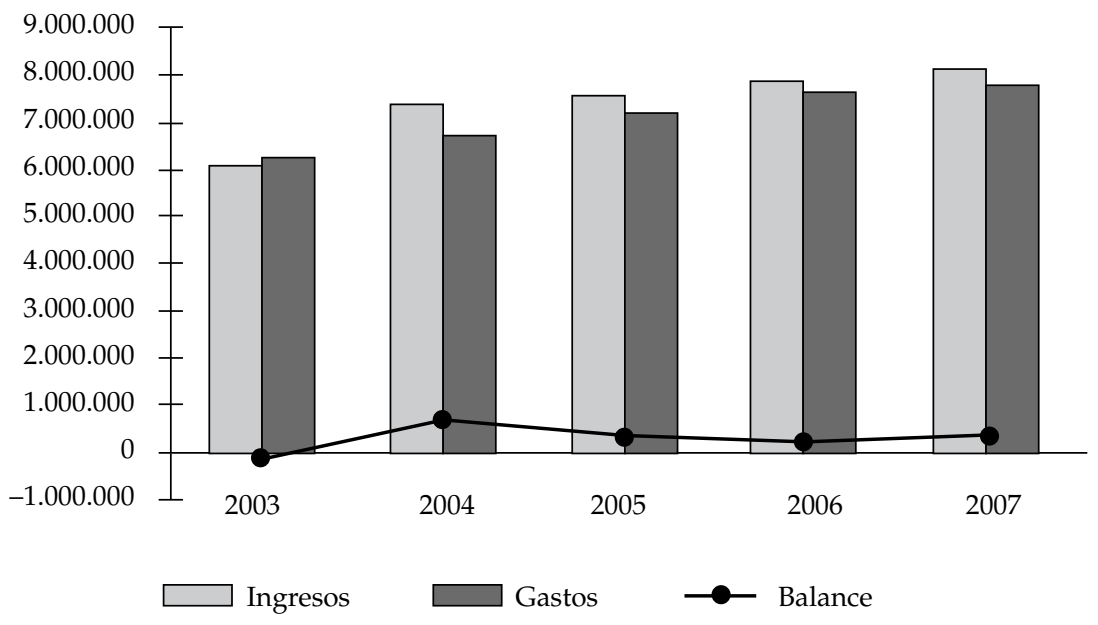




\section{LA OPOSICIÓN EN SU LABERINTO}

Durante el año 2007 la oposición vivió tres momentos políticos. El primero, de convergencia, se extendió de marzo a julio, aproximadamente el segundo, de divergencia, se desarrolló en los meses de junio a agosto, aproximadamente y, el tercero, de recomposición, desde esa fecha hasta fin de año.

El proceso de convergencia fue respuesta a la clara intención del Gobierno de instaurar una hegemonía neostronista a través de una reforma de la Constitución que abriera las puertas a la reelección presidencial. Frenado este intento gracias a la espontánea y multitudinaria reacción de la sociedad civil surge el primer movimiento serio de unificación con vistas a las elecciones de 2008. Este movimiento tiene como figura central a Fernando Lugo, carismático líder que logra aglutinar a todos los sectores de oposición.

El proceso de convergencia llevó a constituir una Concertación Nacional de relativamente fácil parto pero difícil desarrollo. En realidad el problema relativamente menos importante fue el ideológico. Es cierto que la Concertación albergó en su seno desde izquierdistas nostálgicos, especialmente en los partidos más pequeños, hasta admiradores, como Lino Oviedo, el general Park Chung-hee, el dictador surcoreano que gobernó su país de 1961 a 1979. Esto fue lo menos importante, sin embargo, porque en realidad ni los unos pensaban posible hacer realidad el sueño de revivir el pasado ni los otros estaban en condiciones de emular al dictador del sudeste asiático. Los actores más importantes, por otro parte, estaban más interesados en los aspectos pragmáticos de la distribución del poder que en discusiones "teóricas" y además sabían, que en última instancia, por sobre los discursos de uno u otro sector, las políticas del gobierno, de alcanzarlo, oscilarían en torno al centro.

El problema central giró en cambio en torno a la distribución de las candidaturas, pues había sólo dos (presidente y vicepresidente) pero cuatro aspirantes (Lugo, los candidatos del Partido Liberal Radical Auténtico (PLRA), Fadul y, eventualmente, el mismo oviedismo). La candidatura de Lugo, que muchos pensaron se descarrilaría en el camino por una razón legal (impugnación) o práctica (no podría ganar una interna abierta por falta de aparato) finalmente cobró tal fuerza que tirios y troyanos tuvieron que aceptarla como fait accompli pasando luego a disputar sólo la vicepresidencia. Con los oviedistas fuera de juego debido a que el general Oviedo se encontraba cumpliendo su condena por el fallido intento de golpe de Estado de abril de 1996, el campo de la competencia quedó reducido al PLRA y Patria Querida (PQ).

El acuerdo inicial había sido que de no haber consenso en torno a las candidaturas se procediese a la consulta popular, pudiendo ser ésta realizada en forma de una interna abierta o una encuesta. El PLRA, especialmente el precandidato oficialista Carlos Mateo, insistía en la interna abierta a sabiendas de la ventaja que su aparato le otorgaba. $P Q_{\text {"I }}$ por razones obvias, optaba por una encuesta. Y Lugo, lógicamente, en el consenso.

La jugada que inició el proceso de divergencia y desestabilizó el complicado tablero fue el súbito cambio de postura del oficialismo liberal cuando en la Convención del PLRA de 
mediados de año decide resignar esta postura, apoyar formalmente la candidatura de Lugo a la Presidencia, y reclamar la vicepresidencia para el candidato del PLRA. Esta movida no sólo descolocó al candidato de la disidencia liberal, Federico Franco, que tímidamente apoyaba la posición partidaria pero en realidad no se oponía a una encuesta en la que Lugo y Franco, en ese orden, serían los mejor posicionados. El principal golpe lo sufrió $P Q$, pues su candidato, Pedro Fadul, que no alcanzaría la candidatura a la presidencia ni por interna abierta ni por encuesta, aspiraba si, y tenía chance, de ganar la vicepresidencia a los candidatos liberales Franco y Mateo, que irían por separado, y aún quizás, si fuesen unidos, al propio Franco, mejor posicionado entre los liberales.

El razonamiento de los liberales, muy lógico, era no poner en riesgo la candidatura a la vicepresidencia. Al fin de cuentas en las elecciones municipales de finales de 2006 el PLRA había obtenido más de 400.000 votos, mientras que PQ alcanzó apenas un poco más de 70.000. Era evidente también que el PLRA no podía concurrir sin un candidato en la chapa, so pena de exponerse a un proceso de crítica interna susceptible de costarle demasiado caro. Finalmente, aceptar una chapa basada en dos figuras, Lugo y Fadul, sin aparato ni el uno ni el otro, no parecía la estrategia más prudente para la oposición. En síntesis, el candidato a vicepresidente debería proceder del PLRA, que aseguraría a la Concertación contar con un aparato (y a sus propios intereses una adecuada proyección), y el candidato a presidente ser la figura que mas consenso y entusiasmo generaba, Lugo.

Esta movida dejó a Fadul fuera de juego. Sus aliados ofrecieron realizar una encuesta pero sólo para el cargo a presidente e, inmediatamente, pero PQ quería posponerla para octubre y hacerla para ambas candidaturas. Finalmente Fadul abandonó a sus ex aliados alegando que los términos del acuerdo original no se habían cumplido. Si bien esto es cierto, no menos verdadero y mucho más relevante y decisivo es que el panorama político había evolucionado en una dirección desfavorable a sus aspiraciones. Su presencia en la chapa dependía de que Lugo no fuera candidato. Con Lugo candidato era obvio que la otra candidatura debería ser para el PLRA. Aún así, tanto Fadul como PQ hubieran podido jugar un rol relevante en la elección, garantizando el triunfo del cambio, y participando con espíritu crítico en el primer gobierno no Colorado desde 1946. Si Fadul pudo posponer sus aspiraciones en 2003 yendo a una elección que sabía no podía ganar solo, podría haberlas pospuesto en 2008, reservándose para encabezar la lista de Senadores de su partido o para un Ministerio relevante en un nuevo Gobierno. Optó sin embargo por llevar su candidatura hasta el final. ${ }^{8}$ Hoy las encuestas lo sitúan en una posición marginal, con aproximadamente $3 \%$ de las preferencias. Aunque esto mejore es poco probable que con el escenario actual realice una buena elección con lo cual en el mejor de los casos perderá mucho de su capital político y en el peor (si Lugo no gana por la división causada por su candidatura) se expone a la condena de la historia.

Como si todo este movimiento generado entre junio y agosto no hubiese sido suficiente, el Gobierno jugó al final su última carta, el as de la manga. El presidente decidió negociar 
la libertad del general Lino Oviedo. De la noche a la mañana, con inusitada rapidez, un proceso judicial de revisión que llevaba literalmente años se resolvió favorablemente a Oviedo. El Fiscal accedió al pedido de los abogados de Oviedo, la Corte Suprema de Justicia convocó a un número de testigos ex militares, todos declararon no haber "visto" el golpe de Estado, y con la rapidez de un rayo la Corte anuló la sentencia firme y ejecutoriada de 1998, absolvió a Oviedo y el Ejecutivo le devolvió el grado de general en retiro (que le había sido cancelado por haber sido dado de baja deshonrosa). Oviedo recuperó así sus derechos políticos y, ahora candidato, asestó un segundo golpe a la Concertación.

El proceso de divergencia se agudizó. Si las demandas de Fadul no podían razonablemente ser satisfechas, mucho menos podrían ser atendidas sumadas a las ambiciones de Oviedo. En términos de la teoría de Riker (1962) era claro que la "gran coalición", al menos en términos de candidaturas, no podía durar y que el proceso se encaminaba hacia una "coalición ganadora mínima".

En el PLRA se llevaron a cabo las elecciones internas para elección de candidatos a vicepresidente en diciembre. Aunque todas las encuestas daban una amplia ventaja al ex Gobernador de Central Federico Franco, de la disidencia, los resultados sorprendieron a propios y extraños. Franco ganó la presidencia del Partido cómodamente, y sus listas a la conducción del partido y al Senado también se impusieron por amplio margen. Sin embargo, su candidatura a la Vicepresidencia de la República triunfó por un estrechísimo margen de 646 votos, 145.527 a $144.881 .^{9}$

Las acusaciones de fraude fueron y vinieron. Mateo acusó a Franco de haber ganado con votos de muertos en el Distrito de Guarambaré (donde efectivamente se constató que un par de fallecidos aparecieron votando), mientras que Franco levantó un manto de sospecha sobre la "extraña" performance de Mateo y sugirió que detrás estaba la mano del presidente Duarte Frutos que consideraba a Mateo un candidato más fácil de vencer.

\section{EL PARTIDO COLORADO ENTRE LA IMPOSICIÓN Y LA DEFECCIÓN}

Si complicado fue el panorama de la oposición, no lo fue menos el del Partido Colorado. Al principio del mandato del presidente Duarte Frutos hubo una general aceptación de la estrategia de recomponer un grado básico de unidad y disciplina en la acción bajo su liderazgo y de resolver la crisis fiscal del Estado. Pero cuando la estrategia se fue profundizando y surgieron a la superficie aristas desconocidas de la misma, como la reelección, y fracasada ésta, la perpetuación de su liderazgo vicariamente, afloraron también los conflictos.

En líneas generales el Partido se dividió en cuatro bloques. Un bloque, relativamente pequeño, está liderado por el nieto del Dictador Stroessner y los hijos de connotados colaboradores suyos como Mario Abdo Benítez y Carola González Zanotti, y reivindica el stronismo aunque más como glorificación del pasado que como programa de futuro. Lo denominaremos, por 
simplicidad, stronismo de tercera generación. La ex mano derecha del presidente Duarte Frutos y actual presidente interino del Partido Colorado, José Alberto Alderete, formó también su propio grupo cuando el presidente Duarte impuso la candidatura de Blanca Ovelar a la Presidencia de la República. Rompió una relación de más de 20 años reivindicando a la "dirigencia política" del partido, proyecto que, en sus palabras, les inspiró a él y a Duarte Frutos en los orígenes de su movimiento. El tercer frente se constituyó alrededor del ex vicepresidente Luis Castiglioni, que logró el apoyo de la mayoría de los dirigentes del partido y de un número considerable de independientes. Finalmente, el presidente Duarte Frutos estableció su propia fuerza, basada en su liderazgo, la candidatura impuesta de Blanca Ovelar, y la utilización indiscriminada de los recursos del Estado, incluyendo los recursos económicos de las binacionales y nombramientos y remociones en altos cargos como premios o castigos por las preferencias políticas de los funcionarios.

La interna colorada se desarrolló con alta intensidad. El presidente asumió personalmente la jefatura de campaña de su candidata y de hecho fue el personaje central de la misma. Impedido de candidatearse a la reelección, lo hizo como primer candidato a Senador por su partido. Se trata obviamente de una medida inconstitucional por varias razones. Primero, la constitución asigna al presidente saliente el cargo de Senador Vitalicio, con voz pero sin voto.

Este explícito nombramiento le impide competir a la vez por otro cargo. Segundo, todos los altos funcionarios del Estado, ministros y gobernadores, deben renunciar seis meses antes de candidatearse. El presidente no la ha hecho argumentando que en materia de inhabilidades rige sólo lo que está expresamente establecido. Y es verdad que la Constitución no manda explícitamente que el presidente renuncie para candidatearse porque simplemente al asignarle el cargo de Senador Vitalicio no contempla que se candidatee a cargo alguno. Es más, si los ministros deben renunciar para evitar la ventaja del cargo, con cuanta más razón debería hacerlo el presidente, jefe de todos ellos. Una interpretación literal, gramatical, de la normativa constitucional que viola de manera tan frontal el espíritu y el texto de la misma no podría ser aceptada en ninguna instancia judicial independiente. Pero como en el Paraguay la mayoría de los ministros de la Corte responden al presidente, todo observador medianamente avezado coincide en afirmar que cualquier acción de inconstitucionalidad será rechazada.

La interna se realizó el 16 de diciembre. Todas las encuestas daban a la candidatura de Castiglioni considerable ventaja, en algunos casos de hasta 10 puntos. Pero, era obvio que el aparato gubernamental y el dinero iban a jugar un rol fundamental. El oficialismo anticipó que ganaría por 10 puntos, pero a la hora de la verdad y tras quince días de disputas y denuncias de fraude por parte de Castiglioni, el Tribunal Electoral del Partido Colorado (cuyo presidente es funcionario de la Entidad Binacional Itaipú manejada por el Poder Ejecutivo) atribuyó la victoria a Blanca Ovelar por poco más de 4.000 votos.

Según el Tribunal del Partido Colorado, la lista 5 del Movimiento Progresista Colorado (oficialista) con la candidatura de Ovelar obtuvo 366.722 votos, mientras que la lista 4 Vanguardia Colorada (castiglionismo) logró 362.702 votos. La lista de José Alberto Alderete, que contó con el apoyo del stronismo de tercera generación, conquistó 41.058 
votos, mientras que la liderada por Juan Bautista Ibáñez, candidato de los desplazados hijos del ex vicepresidente Argaña, obtuvo 8.892. Se registraron también 16.825 votos en blanco y 14.994 nulos.

Los guarismos que se muestran a continuación en las dos últimas columnas del cuadro indica la fuerte ventaja de Castiglioni en las áreas más pobladas (Asunción, Central y Alto Paraná) y la ventaja que descuenta Ovelar en las regiones más remotas y de menor densidad poblacional.

Tabla 2: Resultados Electorales Oficiales de las Elecciones Internas del Partido Colorado

\begin{tabular}{|c|c|c|c|c|c|c|c|}
\hline Código & Departamento & Habilitados & $\%$ Part. & MRC & $\mathrm{MCI}$ & $\begin{array}{c}\text { Lista } 4 \text { - } \\
\text { MVC }\end{array}$ & $\begin{array}{c}\text { Lista } 5 \text { - } \\
\text { MPC }\end{array}$ \\
\hline 0 & Capital & 224.336 & $50,62 \%$ & 911 & 171 & 60.318 & 44.688 \\
\hline 1 & Concepción & 47.293 & $46,55 \%$ & 73 & 27 & 8.254 & 12.529 \\
\hline 2 & San Pedro & 94.227 & $49,13 \%$ & 1.624 & 82 & 14.428 & 23.487 \\
\hline 3 & Cordillera & 79.34 & $50,79 \%$ & 263 & 49 & 15.120 & 22.205 \\
\hline 4 & Guaira & 66.088 & $54,73 \%$ & 373 & 38 & 14.693 & 17.621 \\
\hline 5 & Caaguazu & 115.226 & $43,99 \%$ & 1.071 & 59 & 14.059 & 29.755 \\
\hline 6 & Caazapa & 53.947 & $57,17 \%$ & 121 & 26 & 13.995 & 12.951 \\
\hline 7 & Itapua & 121.003 & $49,82 \%$ & 445 & 115 & 26.797 & 27.581 \\
\hline 8 & Misiones & 40.059 & $57,29 \%$ & 73 & 36 & 7.689 & 9.450 \\
\hline 9 & Paraguari & 84.025 & $56,18 \%$ & 239 & 48 & 17.773 & 27.042 \\
\hline 10 & Alto Paraná & 164.536 & $48,72 \%$ & 373 & 157 & 47.258 & 28.809 \\
\hline 11 & Central & 425.406 & $43,15 \%$ & 1.676 & 291 & 95.131 & 71.771 \\
\hline 12 & Neembucu & 33.611 & $60,14 \%$ & 1.025 & 38 & 6.275 & 9.028 \\
\hline 13 & Amambay & 29.219 & $49,14 \%$ & 128 & 46 & 5.163 & 5.863 \\
\hline 14 & Canindeyu & 37.353 & $58,69 \%$ & 236 & 70 & 6.136 & 12.811 \\
\hline 15 & Puente Hayes & 30.671 & $54,58 \%$ & 145 & 70 & 7.901 & 7.005 \\
\hline 16 & Alto Paraguay & 5.403 & $67,98 \%$ & 71 & 8 & 1.323 & 1.776 \\
\hline \multirow[t]{2}{*}{17} & Boquerón & 8.071 & $38,55 \%$ & 45 & 14 & 389 & 2.350 \\
\hline & TOTAL & 1.659 .814 & $49,04 \%$ & 8.892 & 1.345 & 362.702 & 366.722 \\
\hline
\end{tabular}

Fuente: Tribunal Electoral Partidario, Asociación Nacional Republicana (Partido Colorado).

El Partido Colorado enfrentará unas elecciones muy difíciles. Es probable que una parte del voto a Castiglioni se fugue a otras candidaturas o simplemente se abstenga de votar. Al mismo tiempo la candidatura de Oviedo es un importante polo de atracción para colorados descontentos que así podrán expresar su rechazo a la candidatura impuesta de Duarte Frutos sin necesariamente votar por la chapa opositora. 
Además, de ganar la candidata del Partido Colorado se pueden generar consecuencias de gobernabilidad graves para el país. En efecto, el escenario actual aparece notablemente similar al que en 1992 y 1993 llevó a Juan Carlos Wasmosy a la presidencia de la mano del General Oviedo. De triunfar la señora Ovelar de Duarte, que no tiene poder político propio ni influencia real sobre los congresistas eventualmente electos, el poder detrás del trono sería en realidad el ex presidente Duarte Frutos que representaría para la misma lo que Oviedo para Wasmosy. Carente del poder que tenía Oviedo en los cuarteles, Duarte Frutos tendría no obstante considerable influencia política como Senador y además asumiría la Presidencia del Partido Colorado - para la cual se postuló inconstitucionalmente en 2006 y a la que pidió permiso luego de asumir- ni bien deje la Presidencia de la República. La señora Ovelar, que ni eligió a su candidato a vicepresidente ni postuló a ningún candidato a cargo electivo alguno, se encontraría así atada de pies y manos a quienes manufacturaron su candidatura.

\section{VI. ¿JAQUE O JAQUE MATE?}

Es imposible finalizar el análisis del año 2007 sin algún ejercicio de interpretación prospectiva, es decir, sin hacer consideraciones acerca del posible resultado de las elecciones previstas para abril 2008. ¿Serán ellas el jaque mate al sistema hegemónico impuesto por el Partido Colorado desde 1946? ¿O representarán otro jaque más, hábilmente eludido con una apropiada movida de piezas que permita la continuidad de la fórmula gatopardista que se ha impuesto en las tres últimas elecciones generales?

En ausencia de un estudio cuantitativo serio y en profundidad, el análisis que sigue se basará en el uso de datos secundarios, especialmente performances electorales recientes, y elementos analíticos derivados de la politología y la sociología política. La mejor manera de empezar es estableciendo primero las expectativas relativamente seguras con respecto a la performance de los partidos tradicionales, que son más estables en el tiempo, y encarar luego el estudio del residuo o voto no tradicional. En este ejercicio se maneja pues una variable cuasi constante, el voto duro o cautivo de los partidos tradicionales, y dos dimensiones variables, el comportamiento del voto blando y el nivel de participación que es lo que determina la magnitud del residuo o voto no tradicional. Puesto que se puede anticipar con razonable aproximación el voto duro o cautivo de los partidos tradicionales en términos absolutos, lo que queda por analizar es el tamaño y comportamiento del voto no tradicional, lo primero dado, como ya hemos señalado, por el nivel de participación y lo segundo por la distribución del mismo entre Lugo y Oviedo, principalmente, y Fadul marginalmente.

Manejaremos tres escenarios de participación, el pesimista que asume una participación de $65 \%$ similar a la tasa de las elecciones de 2003, el moderado que implica una participación del $72,5 \%$ y está basado en el promedio aritmético entre la participación baja del $64,2 \%$ de las elecciones de 2003 y la alta del 80,5\% de las elecciones de 1998 y el optimista basado en la alta participación de las elecciones de 1998 que alcanzó 80,5\%. Usaremos el escenario pesimista como línea de base. 
La votación del Partido Colorado es muy estable, basada en gran medida en un voto cautivo y movilizado por el aparato, y está bastante bien prefigurada en los resultados de sus internas, pues usualmente el Partido Colorado obtiene en las generales un número de votos muy similar al de sus internas. No obstante utilizaremos como predictor del voto colorado el porcentaje obtenido en las elecciones de concejales municipales de fines de 2006 (elecciones de baja participación también, 50\%), cuando el mismo alcanzó 44,50\%. Significativamente, este guarismo extrapolado en números absolutos al escenario de participación baja es 818.070 , cifra prácticamente idéntica al voto total en la interna de diciembre de 2007 que fue 811.193. Se asumirá aquí que el Partido no sufrirá fuga alguna de sus votos, algo muy poco probable, y/o que la que pudiera haber se compensaría con votos nuevos. Este es sin duda el más optimista de los escenarios para el Partido Colorado.

A diferencia del Partido Colorado el PLRA, por su menor capacidad de movilización, consistentemente obtiene en las elecciones nacionales muchos más votos que en sus internas, tendencia que se ha mantenido constante desde el inicio de la transición. ¿Pero cuánto más? A los efectos de este pronóstico tomaremos, como en el caso del Partido Colorado, el resultado de las elecciones municipales (candidaturas a concejales), que fue de $29,82 \%$. Extrapolado a este escenario de participación significa 544.199 votos.

Los datos del Tribunal Superior de Justicia Electoral indican que hay 2.828.245 habilitadas para votar. Una participación del $65 \%$ implicaría que votarían 1.838 .359 personas. Veamos pues los resultados del ejercicio:

Escenario 1: (Pesimista: 65\% de participación)

\begin{tabular}{lll}
\hline Partido Colorado & 818.070 & $44,50 \%$ \\
\hline PLRA & 544.199 & $29,82 \%$ \\
\hline
\end{tabular}

En este escenario ya están "asegurados" el 74,32\% de los votos para los dos partidos tradicionales. Lugo necesitaría sumar un mínimo de 15 puntos porcentuales del total al voto del PLRA para ganar por medio punto, es decir debería conquistar el $60 \%$ del voto no tradicional de aproximadamente $25 \%$.

El segundo escenario, que contempla una tasa de participación intermedia entre la baja de 2003 y la alta de 1998, asume una participación del 72,5\% de participación, es decir votarían 2.050.478 personas. Manteniendo los guarismos absolutos de los partidos tradicionales constantes haremos las modificaciones en los porcentajes que el nuevo nivel de participación indica. El resultado es:

Escenario 2: (Moderado: 72,5\% de participación)

\begin{tabular}{lll}
\hline Partido Colorado & 818.070 & $39,90 \%$ \\
\hline PLRA & 544.199 & $26,54 \%$ \\
\hline
\end{tabular}


En este caso los partidos tradicionales amarran el 66,44\% de los votos. Lugo necesitaría sumar en este escenario por lo menos 14 puntos porcentuales al voto del PLRA, es decir, el $42 \%$ del voto no tradicional que en este caso es ligeramente superior al 33\%. Esto dejaría el 19\% restante a ser distribuido entre Oviedo y Fadul.

El tercer escenario, u optimista, se basa en una participación del $80 \%$ por lo que votarían 2.262.596 personas. Existen considerables similitudes entre la elección de 2008 con la de 1998 en que la oposición iba unida y Oviedo participaba por interpósita persona. La diferencia, por supuesto, es que Oviedo presenta su propia candidatura y lo mismo hace Fadul.

Veamos:

Escenario 3: (Optimista: 80\% de participación)

\begin{tabular}{lll}
\hline Partido Colorado & 818.070 & $36,04 \%$ \\
\hline PLRA & 548.199 & $24,23 \%$ \\
\hline
\end{tabular}

En este escenario los partidos tradicionales se "aseguran" el 60,27 de los votos del total. Para ganar Lugo precisaría sólo aportar 13 puntos porcentuales del total al voto del PLRA, o 33\% del voto no tradicional de poco más de 39\%.

En el cuadro que sigue se resume esta información en términos del mínimo de votos no asegurado por los partidos tradicionales que debe aportar Lugo para ganar. He aquí los guarismos:

Cuadro 3: Mínimos Indispensables para el escenario Lugo gana

\begin{tabular}{lccc}
\hline Escenario & $\begin{array}{c}\text { Puntos } \\
\text { porcentuales } \\
\text { necesarios }\end{array}$ & $\begin{array}{c}\text { Porcentaje del } \\
\text { voto no tradicional } \\
\text { necesario }\end{array}$ & $\begin{array}{c}\text { Número absoluto } \\
\text { de votos } \\
\text { necesarios }\end{array}$ \\
\hline Pesimista & 15 & 60 & 275.754 \\
Moderado & 14 & 42 & 287.067 \\
Optimista & 13 & 33 & 294.138 \\
\hline
\end{tabular}

Hasta aquí las estimaciones pueden considerarse confiables porque se basan en votos estables. ¿Cómo, sin embargo, estimar la distribución del voto no tradicional? El voto de Lugo es por supuesto imposible de calcular en base a datos existentes. Sin embargo es claro que desde 1993 se ha evidenciado un voto independiente que oscila entre el 20 y 25\% del electorado. Es el voto que una vez fue para Guillermo Caballero Vargas (1993), otra vez para Fadul (2003) y podría suponerse que iría en medida importante a Lugo.

El voto de UNACE, en cambio, podría considerarse estable, pues sigue el patrón del voto colorado. Pero como UNACE participó en una sola elección y sin la candidatura de Lino Oviedo este dato es de valor relativo. En 2003 obtuvo 208.391 votos que representó el 
$13,9 \%$ del total. Otra forma de aproximarse a su caudal electoral es observar el resultado de su interna de enero de 2008, donde según sus voceros votaron 90.000 personas o quizás menos pues nunca se publicaron datos completos. ${ }^{10}$ Finalmente, un cálculo basado en premisas más frágiles podría ser deducir del voto de la candidatura Cubas-Argaña (que incluía el factor Oviedo) el voto obtenido por Nicanor Duarte Frutos en 2003, con lo cual se obtendría una aproximación del caudal electoral aportado por Oviedo. En números absolutos se trata de 292.964 votos, número bastante cercano al padrón de afiliados de UNACE que es de 310.610. Es pues razonable especular que el voto de Oviedo oscilaría entre un resultado bajo de 100.000, uno medio de 200.000 y uno alto de 300.000 .

En síntesis, parecería entonces que nunca antes se estuvo tan cerca de un escenario de cambio real. Sin duda no resultará fácil. La campaña electoral recién está empezando y habrá que ver el efecto de la misma y del uso y abuso ilimitados de los recursos del Estado sobre el comportamiento electoral.

Queda también por ver si el Partido Colorado termina impugnando la candidatura de Lugo con el peregrino argumento de que su renuncia como Obispo no es aceptada por el Vaticano y la Constitución impide a los ministros del culto religioso presentarse como candidatos a cargos electivos. Con ello, en un país donde la Constitución consagra la separación entre Estado e iglesia, se pretendería dar al Derecho Canónico estatus de ley positiva y ponerlo incluso por encima de la normativa constitucional. Esta estrategia ha estado presente en el escenario político por meses y sale a superficie de tarde en vez, en parte como ardid electoral para debilitarlo pero también como una posibilidad real.

Si tal cosa no sucediese, el resultado electoral dependería fundamentalmente de dos variables: el nivel de participación (cuanto más alto mayores las chances de cambio) y la distribución del voto no tradicional entre Lugo, Oviedo y en mucho menor medida Fadul.

\section{EPÍLOGO}

Este análisis fue finalizado en enero de 2008. Los resultados de las elecciones del 20 de abril han confirmado el colapso del último sistema de partido hegemónico de América. Aunque no existen disponibles datos finales fidedignos, los siguientes guarismos han sido oficializados en base a un sistema de conteo rápido del Tribunal Electoral sobre el $92 \%$ de las mesas electorales:

$\begin{array}{lr}\text { Lugo } & 41 \% \\ \text { Ovelar } & 31 \% \\ \text { Oviedo } & 22 \% \\ \text { Fadul } & 2 \%\end{array}$

El nivel de participación estimado oscila entre el 66 y el 69\%. 
El voto de Lugo se ajusta al escenario analizado en el texto, con 25 a 27\% aportado por el PLRA y el restante 13 a $14 \%$ por independientes. El voto de Ovelar, que oscilaría alrededor de 600.000, captura sólo el 75\% del voto obtenido por el Partido Colorado en las últimas elecciones municipales o en la propia interna partidaria. La fuga de los votos colorados, producto de la sensación generalizada que el verdadero ganador de la interna colorada fue Castiglioni, favoreció a Oviedo principalmente, y sólo secundariamente a Lugo.

\section{REFERENCIAS}

ABC. 2008. [En línea] <www.abc.com.py>. [Consultas: 4-1-2008; 14-1-2008; 13-3-2008].

ABC. 2007. [En línea] <www.abc.com.py>. [Consultas: 13-12-2007; 17-12-2007].

ABC. 2006. [En línea] <www.abc.com.py>. [Consultas: 21-12-2006].

Abente Brun, Diego. 2007. "Paraguay en la Encrucijada” Revista de Ciencia Política 27 (EE): 221-233.

ABC. 1989. Stronismo, Post-Stronismo, and the Prospects for Democratization in Paraguay. Working Paper 119: Kellogg Institute, University of Notre Dame.

Banco Central del Paraguay. 2007. Informe Económico Preliminar. [En línea] <www.bcp.gov.py>. [Consulta: 4-1-2008]

Carrón, Juan M. 2007. “La emigración paraguaya en el contexto de la globalización”. Ponencia presentada en el $3^{\text {er. }}$ Congreso Paraguayo de Población "Cambio sociodemográfico, urbanización y pobreza". Asunción, noviembre.

Corte Suprema de Justicia. 2008. A.I. $N^{\circ} 17$ del 7 de febrero que hace lugar al pedido de suspensión de los efectos de los artículos 108 y 121 de la Ley $N^{\circ} 3409 / 07$ presentado por la Entidad Itaipú.

La Nación. 2008. [En línea] <www.lanacion.com.py>. [Consulta: 2-1-2008].

La Nación. 2006. [En línea] <www.lanacion.com.py>. [Consulta: 21-12-2006].

Lewis, Paul H. 1980. Paraguay Under Stroessner. Chapel Hill: University of North Carolina Press.

Ministerio de Hacienda. [En línea] <www.ministeriodehacienda.gov.py>.

Portal Paraguayo de Noticias. 2006. [En línea] <www.ppn.com.py>. [Consulta: 25-05-2006].

Riker, William H. 1962. The Theory of Political Coalitions. New Haven: Yale University Press.

Última Hora. 2008. [En línea] <www.ultimahora.com>. [Consulta: 4-02-2008].

U.S. Department of State, Bureau of Democracy, Human Rights, and Labor. 2007. Country Report on Human Rights Practices, 2006. Washington, D.C.

Tribunal Superior de Justicia Electoral. [En línea] <www.tsje.gov.py>.

Diego Abente Brun, Ph.D. en Ciencias Políticas. Director Adjunto del Foro Internacional para Estudios Democráticos de la Fundación Nacional para la Democracia (NED) en Washington, D.C. Fue Profesor Vitalicio de Ciencias Políticas en Miami University, Profesor de Ciencias Políticas en la Universidad Católica, Profesor Invitado de las Universidades Simón Bolívar, Georgetown y George Washington, Fellow del Kellogg Institute de la University of Notre Dame, y autor de numerosos libros y artículos publicados en revistas académicas arbitradas de América Latina, Estados Unidos y Europa. Entre 1993 y 2003 participó activamente en la vida pública. Fue senador y vicepresidente $1^{\circ}$ de la Cámara de Senadores, Embajador ante la OEA, y ministro de Justicia y Trabajo.

(E-mail: diegoa@ned.org) 
